\title{
Building shared situational awareness in surgery through distributed dialog
}

This article was published in the following Dove Press journal:

Journal of Multidisciplinary Healthcare

19 March 2013

Number of times this article has been viewed

\section{Brigid M Gillespie' \\ Karleen Gwinner ${ }^{2}$ \\ Nicole Fairweather ${ }^{3}$ \\ Wendy Chaboyer ${ }^{4}$}

'NHMRC Research Centre for Clinical Excellence in Nursing Interventions for Hospitalised Patients (NCREN) and Research Centre for Clinical and Community Practice Innovation (RCCCPI), Griffith Health Institute, Griffith University, Queensland, ${ }^{2}$ Griffith Centre for Cultural Research, Griffith University, Queensland, ${ }^{3}$ Department of Anaesthesiology, Princess Alexandra Hospital, Queensland, Australia, ${ }^{4}$ Excellence in Nursing Interventions for Hospitalized Patients (NCREN) Research Centre for Clinical and Community Practice INHMRC Centre of Research Innovation (RCCCPI), Griffith Health Institute, Griffith University Queensland, Australia
Correspondence: Brigid M Gillespie National Health and Medical Research Council Centre for Excellence in Nursing Interventions, Research Centre for Clinical and Community Practice Innovation, Griffith Health Institute, Brisbane, Queensland, Australia Tel +61755529718

Fax +61755528526

Email b.gillespie@griffith.edu.au
Background: Failure to convey time-critical information to team members during surgery diminishes members' perception of the dynamic information relevant to their task, and compromises shared situational awareness. This research reports the dialog around clinical decisions made by team members in the time-pressured and high-risk context of surgery, and the impact of these communications on shared situational awareness.

Methods: Fieldwork methods were used to capture the dynamic integration of individual and situational elements in surgery that provided the backdrop for clinical decisions. Nineteen semistructured interviews were performed with 24 participants from anesthesia, surgery, and nursing in the operating rooms of a large metropolitan hospital in Queensland, Australia. Thematic analysis was used.

Results: The domain "coordinating decisions in surgery" was generated from textual data. Within this domain, three themes illustrated the dialog of clinical decisions, ie, synchronizing and strategizing actions, sharing local knowledge, and planning contingency decisions based on priority.

Conclusion: Strategies used to convey decisions that enhanced shared situational awareness included the use of "self-talk", closed-loop communications, and "overhearing" conversations that occurred at the operating table. Behaviors that compromised a team's shared situational awareness included tunneling and fixating on one aspect of the situation.

Keywords: shared situational awareness, surgery, distributed dialog

\section{Introduction}

Surgery is an integral component of global health care, with an estimated 234 million operations being performed annualy worldwide. ${ }^{1}$ However, the operating room is imperiled by the threat of adverse events, many of which are related to communication. Insufficient or ineffective communication is the most frequent cause of adverse events across all areas of health care, culminating in harms that range from delays in treatment to medication errors to wrong site surgery. ${ }^{2-4}$ A systematic review of adverse event studies revealed that $41 \%$ of all hospital adverse events occurred in the high-risk milieu of the operating room ${ }^{5}$ and a retrospective audit of patient medical records suggested that $50 \%$ of identified events were preventable. ${ }^{6}$ Specifically, communication and teamwork failures in surgery were identified as a contributing factor in $38 \%$ of incidents in nonemergency procedures and could impact in up to $59 \%$ of incidents in emergency cases. ${ }^{3}$ While the imperative to avoid communication errors in the operating room is widely promulgated in patient safety discourse, progress has been slow. 7,8 Ambiguities, silences, or omissions in conveying clinical decisions to other members 
of the surgical team during the intraoperative period impact on team cohesion, hamper performance, and can contribute to procedural error. However, there is a paucity of research describing the information rendered in creating the dialog around clinical decisions among members of the surgical teams. The aim of this observational study was to describe the strategies used to communicate decisions during surgery and the ways in which this dialog creates or compromises shared situational awareness.

The concept of situational awareness was first described in aviation to explain the perceptual skills needed for the success of fighter pilots. ${ }^{9}$ Situational awareness refers to an individual's ability to maintain the "big picture" in a dynamically changing environment. ${ }^{10}$ Endsley ${ }^{11}$ proposed three levels of situational awareness based on perception, comprehension and understanding, and projection. Put simply, situational awareness is characterized by three questions, "Where have we come from?", "Where are we now?", and "Where are we going?". ${ }^{12}$ Within the three levels of situational awareness, four key factors in Endsley's ${ }^{11}$ model include: the role of goals in directing attention to determining the significance of perceived information; the role of expectations informed by current perceptions of the situation; different methods of information processing (eg, analytic and intuitive decisionmaking) to achieve situational awareness; and the salience of feedback in maintaining situational awareness. As such, situational awareness relies on differentiating situations that may appear to be similar to determine the scope of the situation. ${ }^{13}$ Each situation has its own associated set of possible futures, depending on the actions of the individual. Situations are classified on a continuum ranging from clear, routine, and easily managed at one extreme, to confusing and dangerous, requiring particular skills and expertise. ${ }^{13}$

In surgery, teams of surgeons, anesthetists, nurses, and technicians work together on tasks towards a common end goal. While situational awareness is critical to the performance of individuals, it is also crucial to the performance of the team as a collective. ${ }^{14}$ Shared situational awareness is when members have an appreciation of each other's activities and share common perspectives of events and goals. ${ }^{15}$ Shared situational awareness suggests that during team activities, situational awareness overlaps and individuals need to perceive, comprehend, and project situational awareness components specific to their role in the team. Successful performance consequently requires that team members have a good situational awareness of their specific components and also similar situational awareness for shared components. ${ }^{10,16}$ Specifically, shared situational awareness requires team members to have an understanding of the type of information needed by others, knowledge of the devices used to distribute situational awareness (eg, shared visual displays), shared team processes to facilitate sharing of relevant information (eg, communication, coordination, cooperation), and shared mechanisms, ie, a shared mental model. ${ }^{10,14}$ A shared mental model enables team members to adapt swiftly and coordinate their actions based on their underlying knowledge and understanding of each other and of the present situation. ${ }^{16}$ In high performance teams, when a significant change in the situation is detected, a more focused assessment takes place, rendering a proactive rather than reactive response to expected and unexpected events. ${ }^{14}$

While there is a need for teams to cultivate shared situational awareness through effective communication, individual members often experience disconnections in situational awareness. ${ }^{14}$ Disconnections in situational awareness can occur when information is not passed between members in a clear and concise manner. In addition, when the information is passed on to others, team members may interpret the information differently, based on dissimilar mental models. ${ }^{14,16}$ Finally, even when team members share a similar comprehension of the current situation, they may have disparate projections of what is likely to happen. Thus, the different mental models that team members possess have a bearing on the predictions they make. ${ }^{14}$ Communicating consequential information during surgery ensures that members are "on the same page" and enhances coordination.

Previous human factors research has examined the broad domains of situational awareness. ${ }^{10-13}$ However, there is limited research that describes the dialog of clinical decisions communicated to team members and how this enhances or compromises the team's shared situational awareness. Studies of coordinating decision-making are rare, and have tended to focus on the technical aspects of surgical procedures, ${ }^{17}$ while others have explicitly focused on the role of the surgeon ${ }^{17-19}$ or the scrub nurse, ${ }^{20}$ with little attention given to the surgical team as a collective. In the operating room, team members often have to make decisions under time pressure, increasing risk, and when unforeseen conditions and unanticipated problems emerge. This is not only pertinent to emergency surgery, because capricious situations requiring new decisions and/or a change of plan may also occur during elective surgery. This study is important because it identifies strategies that may help team members build shared situational awareness. It is useful to understand the dialog that underpins decision-making in surgery. The strategies used to support this dialog build situational awareness and allow members 
to recognize aspects of the situation that may pose a threat to safety and ensure that, if required, other team members are able to respond in a timely and appropriate way.

\section{Materials and methods}

A qualitative study design based on fieldwork methods using participant observations and semistructured interviews allowed us to describe the content and context of dialog around decisions, and the ways in which this enhances or hampers team members' ability to build shared situational awareness.

\section{Setting and sample}

This study was conducted in the operating department of a large metropolitan teaching hospital that caters for all surgical specialties, except for gynecology, obstetrics, and pediatrics, in Queensland, Australia. At the time of this study, the operating suite had 22 commissioned operating rooms and performed over 18,000 operative procedures annually. The department was staffed by over 250 health care professionals who held surgical, medical, nursing, and ancillary roles and provided around-the-clock coverage.

In this facility, the team consisted of surgical and anesthetic consultants and their trainees and residents, and registered and/or enrolled nurses practicing in scrub, scout, or anesthetic roles (in this facility, registered and licensed practical nurses were employed to perform scrub [instrument]), scout [circulating], and anesthesia assistance roles. Participants were purposively selected, ${ }^{21}$ based on their ability to render useful insights on this subject, and consisted of members from surgery, anesthetics, and nursing. The choice of surgical procedures was purposive and participants were drawn from interdisciplinary groups who worked across 10 surgical specialties (ie, vascular, cardiac, general, orthopedic, ophthalmology, otolaryngology, faciomaxillary, plastics, urology, and neurosurgery). Maximum variation sampling ${ }^{21}$ was used to capture diverse characteristics in relation to different surgical specialties, divergent levels of clinical experience, and subspecialty roles. By comparing the responses and experiences of participants against each other, discrete but important differences were uncovered ${ }^{21}$ about teamwork and decision-making practices in surgery.

\section{Data collection}

Fieldwork progressed over 6 months and data were collected during 2009-2010. Methods included a reflective diary, participant observation, field notes, and focused interviews. The authenticity of a field study relies on the experience and expertise of the observer. The first-named author (BG) was trained in human factors research, had extensive experience as an operating room nurse, and performed all field work observations and interviews. BG kept a diary and journaled her perceptions prior to, during, and after field work to reduce the propensity to accept any internalized, taken-for-granted assumptions about context. ${ }^{22}$ During fieldwork, BG was located away from the operating table, with each team member and all entrances to the room in view.

Semistructured interviews were used to elicit participant perceptions ${ }^{23}$ of teamwork practices and decision-making in surgery. Participants also validated information gathered through other sources, including observations and statements made by other team members. Interviews lasted 25-60 minutes, and were conducted at the discretion of the participant in a room away from the main work area. Based on their personal preference, participants were either interviewed in groups or individually. A general interview guide was used and topics covered explored issues surrounding the contextual factors that fostered or threatened effective interdisciplinary teamwork. As the fieldwork progressed, questions varied and new questions were asked based on emergent findings. For example, the majority of medical participants described communications that occurred around clinical decision-making. Therefore, additional questions, ie, "How did you know to make the decision?", "What information did you need?", and "How was this information conveyed to other team members?", were asked in subsequent interviews. Data saturation was evident as the analysis progressed and precipitated an end point to data collection when no new information was revealed. Prior to commencing the interviews, demographic data were collected in respect to participant age, years of experience, and professional role. All interviews were digitally recorded for later transcription.

\section{Ethics}

Ethics approval was given by the human research ethics committees of the hospital and the university. No members of staff were interviewed or observed without informed written consent being obtained. During the study period, consent was renegotiated and participants were informed about their right to withdraw at any time.

\section{Analysis}

The analysis was performed using the transcribed field notes and interviews in an iterative manner that involved open coding and categorizing to develop themes. ${ }^{23}$ Codes were 
developed based on topics raised by participants in relation to teamwork practices and were subsequently categorized based on their similarities and differences. Thematic analysis involved a process of breaking down, examining, comparing, and conceptualizing data to enable recognition of emerging patterns to identify major themes. ${ }^{24}$ Theme identification reflected recurring events and dialog with similar content, both within and across the textual data. The themes extracted and their corresponding explanatory data were crosschecked among the research team to ensure consensus. In labeling the themes, a thick description was used to indicate the context from which they were drawn. During analysis, meetings were held with the coresearchers to discuss and reconsider emerging themes and subthemes.

\section{Rigor}

In establishing trustworthiness, the tenets of auditability, triangulation, and transferability were considered. ${ }^{25,26}$ All members of the research team were involved in data analysis in order to identify irregularities and establish consistency. Once complete, the preliminary findings were taken back to participants for clarification and confirmation. Memos connecting emerging findings to pieces of verbatimsupported emergent themes, and demonstrated an audit trail. Triangulation was achieved through use of multiple data sources, which allowed a broad range of issues to be crosschecked, achieving convergent validity. ${ }^{26}$ There was purposive selection of a wide range of participants, based on maximum variation ${ }^{21}$ which enabled a comprehensive overview of the phenomena under study, so there may be conceptual transference to other similar settings.

\section{Results}

Over 500 hours of observations were recorded involving 39 surgeons, 26 anesthetists, 63 nurses, and 15 ancillary staff across 10 surgical specialties. During the 6-month observational period, 160 surgical procedures were observed. In total, 16 individual and three group interviews were conducted with 24 participants (Table 1). Participant age ranged between 23 and 66 years, and their years of clinical experience from 12 months to 40 years.

The domain of coordinating decision-making in surgery encompassed the complexity of the surgery, the condition of the patient, knowledge of the task, and the skills and abilities of other team members. "Coordination" concerned the deliberate effort at managing the task through explicit distributed communication that allowed team members to build their situational awareness. "Clinical decision-making"
Table I Number of participants interviewed, method of interview, and specialty

\begin{tabular}{lll}
\hline Participants $(\mathbf{n})$ & Method of interview & Specialty \\
\hline 13 nurses & I group & Cardiac \\
& 2 groups & All other specialties \\
& 5 individual \\
6 anesthetists & 6 individual & Cardiac \\
& & All other specialties \\
5 surgeons & 5 individual & $\begin{array}{l}\text { Vascular } \\
\text { General } \\
\end{array}$ \\
& & $\begin{array}{l}\text { Orthopedic } \\
\text { Plastics }\end{array}$ \\
& & Neurosurgery \\
Total 24 participants & 19 interviews & \\
\hline
\end{tabular}

entailed identifying and considering options, selecting and communicating options, and re-evaluating and reviewing decisions based on these options. Subsumed within this domain were three interlinking themes, ie, synchronizing actions and strategizing to adapt, sharing local knowledge, and planning contingency decisions based on priority. Table 2 shows each of these themes and their supporting subthemes. What follows is an interpretative account of each theme with supporting verbatim.

\section{Synchronizing actions and strategizing to adapt}

The theme of synchronizing actions and strategizing to adapt was underpinned by the capacity of team members to communicate decisions in the management and timing of individual and team tasks in an explicit manner. "Synchronizing" and "strategizing" incorporated communicating a course of action based on team members' level of situational awareness. The use of open explicit dialog around decisions facilitated distributed situational awareness, allowing participants to respond appropriately to the cues provided by others. However, there were also occasions when participants described the need to consciously focus on the task at hand, even if this at times culminated in loss of the peripheral information sources needed to inform such decisions. For instance, anesthetist participants particularly emphasized the use of algorithms as a decision-making strategy to deal with an anesthetic emergency:

\footnotetext{
"You have a series of algorithms which you go through but because you have a reasonable idea of what is going wrong, you step into the algorithm half way down it instead of starting at the top and then because you are wrong you get the wrong result ... You get fixated and finish up thinking you are quite sure you are getting evidence but then the
} 
Table 2 Domain themes and their supporting subthemes

\begin{tabular}{|c|c|}
\hline Theme & Supporting subtheme \\
\hline $\begin{array}{l}\text { Synchronizing actions } \\
\text { and strategizing to adapt }\end{array}$ & $\begin{array}{l}\text { - Coping with competing demands } \\
\text { - Deviating from procedural flow } \\
\text { - Rethinking the procedure } \\
\text { - Leading } \\
\text { - Taking cues } \\
\text { - Discussing concerns } \\
\text { - Seeking correspondence } \\
\text { - Recognizing errors } \\
\text { - Shutting down }\end{array}$ \\
\hline Sharing local knowledge & $\begin{array}{l}\text { - Knowing the patient } \\
\text { - Knowing the procedure } \\
\text { - Knowing each other } \\
\text { - Following the procedure } \\
\text { - Being on the same page } \\
\text { - Relying on previous knowledge } \\
\text { - Und experience }\end{array}$ \\
\hline $\begin{array}{l}\text { Planning contingency } \\
\text { decisions based on priority }\end{array}$ & $\begin{array}{l}\text { - Deciding what is urgent } \\
\text { - Thinking one's way through } \\
\text { - Pressing the emergency button } \\
\text { - Modifying activity/procedure } \\
\text { - Getting stuck } \\
\text { - Making a judgment call } \\
\text { - Making high-risk clinical decisions }\end{array}$ \\
\hline
\end{tabular}

lights go on and you go 'oh no, I was wrong'." (Consultant anesthetist, interview 7)

This anesthetist's failures in perception and comprehension of the situation resulted in an incomplete mental model. Consequently, an over-reliance on a set of prescribed steps stymied this participant's ability to resolve the problem at hand because he was locked into an incorrect picture of the situation. It was only when an adverse event occurred that the anesthetist realized his comprehension of the situation was flawed. Another participant revealed that when an error in judgment occurred, the ability to process information from other sources rapidly diminished:

"When you have a situation where something does go unexpectedly wrong or you do something that has a bad outcome and things are deteriorating quickly then you feel the anxiety rising, you focus, you tunnel, you really are trying to think clearly but because there is all this 'oh my God, oh my God what have I done?', when things go wrong unexpectedly because you have done something, you are so focused on that that you often forget about the peripherals. You almost shut down ..." (Consultant anesthetist, interview 6)

The distress of having an unfavorable outcome had a debilitating impact on this participant's situational awareness, causing her to "shut down", thus limiting her capacity to convey decisions to others at the height of a crisis situation.

Decisions around controlling the tempo of surgery during crucial stages were described:

"I deliberately, consciously slow down, when I am doing a hepatectomy; just near the end there is a rush, the organ is hanging on by a slither, you have a huge amount of liver coming out, your big hand hanging onto it and the temptation is to draw on it and accelerate the process which increases a vascular injury so it is at that point where I say publically to everyone 'I am going to slow down because I am getting to the critical last little step'. I talk people through what I am doing and am talking myself through it ... I like to have as much correspondence as possible at that critical time." (Consultant surgeon, interview 16)

The overt act of "talking through" the delicate concluding stages of the procedure enhanced team situational awareness because it served a dual purpose: it permitted the surgeon to keep abreast of the situation, achieve coherence and agreement with other team members on the goals of the task; and also allowed the surgeon to be more accessible to the assistance, suggestions, advice or questions from others. In contrast, junior surgeons tended to fixate on the technical problem during critical periods of the operation, and reduced the exchange of information to a minimum during that time. Only information related to perceptions of the status and dynamic components of the immediate situation, ie, level 1 situational awareness, ${ }^{11}$ was communicated to others under the assumption that they would reach similar projections as the operating surgeon.

Scrub nurses articulated the importance of organizing the layout of their sterile work area in a way that it allowed them to place their hand directly onto the instrument without looking away from the operative field. Experienced scrub nurses focused on the operative field and listened for conversational exchanges between consultant surgeons and their trainees to learn of changes to the operative plan. These behaviors increased nurses' levels of situational awareness and they were able to pre-empt the surgeon's needs and "think ahead" to ensure that the operation progressed smoothly. An experienced nurse stated that her decisions on the selection of instrument to pass to the surgeon at any given stage of the operation were essentially governed by her understanding and conception of "what's happening now, and what can possibly go wrong".

\section{Sharing local knowledge}

The second theme, ie, sharing local knowledge, encompassed context-specific understandings of individuals and 
the situation as a whole, as well as its many facets, and the ways such understandings contributed to the communication of decisions to build shared situational awareness. "Local knowledge" referred to team members' awareness of nuances and practices of individuals and their roles in the context of different surgical specialties. Accordingly, team members' awareness of individual capabilities, the ability of members to cohese as a collective, the condition of the patient, and the status of the procedure being performed, and the environment (equipment, position of the patient, room layout), not only influenced the decisions made, but also how the dialog was constructed and conveyed.

Shared situational awareness in cardiac surgery was fostered by the deliberate use of closed-loop communication to convey that the steps taken in making a decision were purposeful, logical, and deliberate. In the cardiac room, tasks around perfusion and cardioplegia were managed by the perfusionist and cardiac surgeon through a series of sequenced actions and minicommunications with the aim of identifying the cause of the problem and returning the patient's system to a more normal, desirable state. ${ }^{27}$ In the context of this study, a perfusionist is an anesthesiologist who has received additional specialist training in cardiac perfusion and cardioplegia. Coordination of actions was frequently heralded by a deliberate annunciation of steps taken by the perfusionist and surgeon, making explicit their expectations based on the current situation:

\footnotetext{
"In cardiac anesthesia and perfusion the rule is that when you are given an instruction or asked to do something, you confirm verbally that that is what you are doing ... Cardiac is very regimented, there is one way of doing things and that is it. I anaesthetize and perfuse differently and behave differently in cardiac than I do in general theaters." (Consultant anesthetist, interview 12)
}

In the cardiac room, attention to the orchestration of actions allowed both the surgeon and perfusionist to recognize urgency and use appropriate recovery methods to manage deviations from expected events. The dialog around decisions in the cardiac room was in stark contrast with the way decisions were communicated among team members in other surgical specialties. For example, conveying or confirming decisions about a change in case requirements (eg, impending need for a microscope) or changes in the order of patients listed for a morning or afternoon session based on procedural complexity and the availability of consultant surgical expertise, appeared to be made independently, without consultation with other key personnel in the room. In this instance, the disparate mental models that members possessed likely influenced their comprehension and the projections they made.

Although procedures may be booked as elective or "routine", cardiovascular fluctuations during surgery, and variations in the acuity of the patient and their surgical anatomy, influenced clinical decisions concerning the approach and ongoing management of these cases:

\footnotetext{
"There will be lots of little subtleties that have gone on in raising a flap, transposing a flap, in doing a resection, whatever. Those little changes that have occurred throughout the operation, it is still the same operation but it is different from the way you might have done it last week. If you haven't seen all those things it might affect the way you communicate in the operation." (Trainee surgeon, interview 8)
}

For this participant, prospectively communicating decisions based on changes in case needs and/or patient status alleviated the trepidation associated with potential or projected changes in tissue vascularity that frequently occurred during microsurgery. This behavior also served to inform other team members, thus building shared situational awareness. The observer witnessed instances when surgeons requested instruments and additional equipment to be taken onto the table in anticipation that they would be needed, albeit that they were not always used. Giving this information well in advance permitted scrub nurses to prepare for probable challenges or changes. In contrast, delays, silences, or omissions in communicating imminent case needs to other team members based on a change in plan or approach culminated in members of the surgical subteam waiting at the operating table for an additional piece of equipment. As such, procedural disruptions occurred when the scout nurse left the room to retrieve a particular instrument or tray. Clearly, disparate levels of access to vital information impacted on team members' situational awareness, leading to different projections of what was likely to occur in the near future.

Nurse participants described behaviors that augmented their situational awareness. For instance, the importance of being focused throughout the operation in respect to careful listening and intent watching; thus, knowing and understanding the normal sequences of the procedure was considered essential in gleaning useful information about the progress of surgery. In selected instances, the scrub nurse became the "gatekeeper" to the surgeon, ${ }^{28}$ vetting information flow based on their assessment of the status of the procedure:

\footnotetext{
"The scrub nurse is the best person to make the judgment as to when to ask for things because they know what part of
} 
the operation is the most critical or noncritical." (Registered nurse, interview 2)

Determining what information should be filtered through to the surgeon encompassed several factors, ie, experienced scrub nurses possessed good technical and scientific knowledge of the procedure being performed, an appreciation of its intricate and difficult stages, and had an understanding of any subtle changes in the demeanor of individual surgeons. Both scrub and scout nurses were less likely to interrupt when the procedure was at a stage that required intense concentration by the surgeon, such as during the clipping of a cerebral aneurysm in neurosurgery. Scout nurses also judged the urgency of incoming messages when answering cell phones or by questioning the approaching outsider regarding the details of the message. More experienced nurses intuitively discerned the surgeon's mood and used this knowledge to inform decisions about how they would interact with them, altering their approach as necessary.

\section{Planning contingency decisions based on priority}

The final theme, ie, planning contingency decisions based on priority, illustrated the unpredictable nature of a myriad of clinical scenarios and the dilemmas confronting team members when making decisions about the urgency of events. "Contingency decisions" referred to timely and explicit dialog based on a change of plan or priority, and thus fostered shared situational awareness. The majority of medical participants affirmed that "judgment calls" involved making decisions around priorities of care; the essence of which was captured in this comment by one of the trainee surgeons:

"... an emergency is by its nature very different than an elective case. So the goals are different."

Yet decisions based on the priorities of patient care were not always mutually agreed upon, or discussed.

"From a basis of not having seen the patient, the surgeon has booked the case and they will give it a 'category 1' being the most urgent and ' 5 ' being almost elective, but they often incorrectly categorize their patients so they appear to be more urgent so they are done in hours or at a convenient time or will say 'this is an urgent case but we are not available until 5 pm'. We end up saying 'that's rubbish you are not doing that or okay this case needs to go before that case'. We often have to bump the orthopods out of theater to do an appendix or something." (Consultant anesthetist, interview 7)
This scenario was common, ie, the triaging anesthetist's decisions were informed by the urgency of the procedure and how "sick" the patient was. Floor coordinating nurses also made decisions around assigning priority when elective lists would over-run. The priority given to which listed cases would be cancelled was driven by time constraints and limited staffing to cover list over-runs. Such decisions were also informed by the coordinating nurse's knowledge of the procedure, and how long it would take individual surgeons to complete the procedure within the remainder of the session. The coordinating nurse would enter the room and, in a low tone, speak quietly to the anesthetist, seeking agreement and support for the decision to cancel the case. Once agreement had been reached, the decision was subsequently communicated to the attending surgeon. Conversely, there were times when semielective or emergent cases could be booked or added to a surgical list; accordingly, both the surgeon and the anesthetist in the room were approached simultaneously by the coordinating nurse.

In assessing the situation and prioritizing actions, surgeons described "threats" in terms of the patient (atypical anatomy, adhesions from previous surgery, unexpected findings, excessive blood loss), the task (visibility or access problems, time pressure), and the team (inexperience, unfamiliarity with the procedure). "Risk" was considered in terms of potential harm to the patient, the surgeon, other team members, and the organization. ${ }^{19}$ The imperative to adapt or change in crisis situations and manage risk was patently illustrated when a surgeon participant abandoned his original operative plan because the patient's condition was obviously deteriorating. In articulating the need to change pace and shift strategies during surgery, he eloquently described decision-making that was based on a hypothesized outcome:

"I said to her (scrub nurse), 'We need to get this patient closed' (patient's wound incision) because we did switch our strategy. At a certain stage in the operation it switches from conventional surgery to what we call 'damage control' where you stop all the bleeding, control the bowel contamination, but as soon as you have done that, you hurry up, pack the patient and send them quickly to ICU. So I had to say 'we are changing the pace now'. It is a really important change in strategy and this all comes from trauma protocols." (Consultant surgeon, interview 16)

Based on the surgeon's assessment of the current situation, he believed that there was little choice because of the tenuous balance of risk involved in unnecessarily prolonging 
this critical surgery. The priority was to prevent further deterioration and deal with life-threatening injuries rather than to restore function, reconstruct tissues, or improve perfusion. While other options may have been momentarily considered, the patient's deteriorating condition meant that the definitive course of action, with apparently little conscious deliberation, was conveyed to the scrub nurse. During decision-making, the experience of the surgeon and his or her ability to process information efficiently from other sources informed the decision. The delivery of explicit and timely dialog supplemented the mental model of other team members, building shared situational awareness, assisting members in preparing for a change of plan. In many instances, participants had to retrieve information regularly from multiple sources to make informed decisions, but often only had access to partial information that was relevant to a successful outcome. Consequently, on occasions when "bigger picture" information was not volunteered, disparities in the perceived level of risk were further compounded:

“If you are going into a case, a situation where you don't perceive there is great risk, the surgeon may perceive there is great risk but that hasn't been communicated. We go into a situation where we are dealing with someone who is a great risk but they are only operating on a toe nail ... once you have started if something is not quite right or if perhaps you are starting on a path the rest of the team mightn't know." (Consultant anesthetist, interview 3)

\section{Discussion}

The findings herein suggest that "coordinating decisionmaking in surgery" encompassed synchronizing and strategizing actions, sharing local knowledge, and planning and prioritizing contingency decisions. Explicit, distributed, and timely communication is crucial in facilitating the exchange of information for cohesive teamwork, as tasks and roles are deconstructed, performed and reconstructed in their entirety. ${ }^{29}$ In this study, efficient communication of clinical decisions heightened team members' awareness of actual or potential problems, building shared situational awareness and assisting them to respond appropriately.

The first theme, ie, synchronizing and strategizing actions, described behaviors associated with decisions around focusing on the immediate task, controlling the pace of surgery through "self-talk", and "thinking ahead" by the surgeon. At critical junctures of the procedure, the effects of stress were evidenced in the ways that the communication patterns of surgeons and anesthetists changed in the absence of discussing crucial "next step" decisions. Some junior doctors "shut down" and, instead, compensated by fixating on the technicalities of the task. Alternatively, more experienced surgeons were able to sustain open dialog with others, thus explicitly coordinating tasks. The use of "self-talk" appeared to be pivotal in maintaining shared situational awareness, and also enabled surgeons to manage their stress levels. "Self-talk" is described as the purposeful internal conversation that motivates an individual's performance. The ongoing dialog assists others to maintain vigilance, particularly during high tempo periods, because less information needs to be communicated relative to what is already mutually known and understood. ${ }^{30}$

Scrub nurses' behavior of "overhearing" conversations at the operating table heightened their situational awareness. Overhearing conversations may be likened to using "voice loops" where individuals or groups obtain direct, real-time updates for a particular decision-maker. ${ }^{31} \mathrm{~A}$ "voice loop" is a real-time auditory prompt that enables distributed translation of information among individuals in close physical proximity. Voice loops, although uncommon in health care environments, are increasingly being recognized as an effective coordinating tool in high dependency settings. ${ }^{31}$ In our study, the act of listening in enabled scrub nurses to coordinate their activities, anticipate unexpected events, plan contingency responses, understand the rationale for nonroutine requests, and note fluctuations in voice tone that may signal an escalation in activity. Nurses were thus able to participate actively in decision-making around coordination of personnel and equipment needs on selected occasions. Our findings extend recent research which suggests that nurses have limited scope to make decisions in the operating room milieu. ${ }^{20}$ While nurses within surgical teams are not responsible for making "life and death" decisions in surgery, they are privy to many clinical decisions, and are thus tasked with communicating decisions about the synchronization of activities to others, and act as conduits of information. Concordant with recent research, ${ }^{20}$ the nurses in this study used situational awareness to build a mental model by gathering cues presented in the environment and integrating information from an array of sources, much of which was based on their familiarity and experience with the surgeon and the procedure.

The second theme, ie, sharing local knowledge, illustrated the importance of previous exposure to similar situations, understanding other team members' capabilities, and the ways in which their roles and tasks intersected to meet shared team goals and expectations, and the use of closed-loop 
communication. Complete shared situational awareness is often impossible, but using closed-loop communication guides effective coordination, and its absence diminishes team members' ability to develop a shared mental model. ${ }^{32}$ In the cardiac room, the surgeon and the perfusionist have limited access to information that is relevant to a successful outcome. ${ }^{27}$ Both the surgeon and perfusionist perform distinct tasks that demand unique expertise, yet these actions require careful orchestration if they are to meet the shared goals of surgery. Situational awareness arises out of the process of using closed-loop communication in cardiac surgery because these structured minicommunications establish the current state of the patient's system and the sequencing of actions needed to perform tasks. ${ }^{27}$

Nurse participants in our study tended to make intuitive decisions based on experiential knowledge, and this accords with previous research. ${ }^{33}$ Nurses' decisions centered on coordinating case requirements, booking and cancelling listed procedures, and choosing the most appropriate way to approach different surgeons according to their demeanor and situation. Nurses filtered information that reached the surgeon, thus permitting surgeons to remain disconnected and separate from their broader environment, and allowing surgeons to concentrate on "the act of surgery", which are notions identified in earlier critical discourse. 28 "Gating" information flow appeared to reduce the incidence of interruptions which have been previously identified as contributing to clinical error. ${ }^{34,35}$ However, vetting information may also have undesirable, unintended effects, ie, selectively communicating information to surgeons that nurses deemed as nonurgent may have contributed to an error elsewhere. Essentially, in this situation, it is likely difficult for nurses to make distinctions between urgent and nonurgent messages because they do not necessarily know the context or the vital background information.

The final theme illustrated team behaviors around planning and prioritizing contingency decisions. The level to which participants were able to build situational awareness was determined by their ability to foresee, manage, and communicate their assessment of potential situational risks. Most medical participants in our study considered clinical decisionmaking in terms of patient-related or task-related risks, and echoes findings elsewhere. ${ }^{18,19}$ Senior surgeons illustrated situations where emergency decisions were underpinned by a judgment of likelihood or an estimation of risk, ie, decisions that are likely to be made on the basis of intuition and experience. ${ }^{19}$ However, it appeared that some participants had limited access to all relevant information regarding risk, limiting their ability to respond as expected. When decisions were enacted in emergency and time-pressured situations, distributed communication about the level of risk was, at times, constrained. In other cases, differing comprehensions of the current situation (levels 1 and 2 situational awareness) ) $^{32}$ secondary to disagreements about the patient's level of risk, may lead to an unfavorable outcome. Situation assessment requires cognitive processing, and therefore contributes to workload. ${ }^{29}$ Conceivably, situational awareness suffers because of competing demands, and even striking events can be missed because they are outside the individual's sphere of attention at that time.

\section{Strengths and limitations}

This study has a number of strengths, but we also recognize that several limitations exist. First, the single locale of the study setting limits the extent to which its findings may be generalized because the staff working in this hospital may be different in some way. Despite this, there was representation across disciplines, permitting illumination of diverse professional perspectives and enhancing conceptual transferability. ${ }^{21}$ Further, our findings are consistent with those of several other studies of communication and decisionmaking in surgery. ${ }^{18-20}$ Second, there are innate complexities in examining a context with which the observer has a high degree of familiarity. Still, preconceived assumptions were challenged by diarizing field experiences and writing memos to enhance objectivity and professional judgment. ${ }^{22}$ As such, the effects of bias during data collection, interpretation, and description were minimized. Finally, the differing methods used for some of the nurse participants may have given rise to different dynamics during interviews. However, similar issues were explored and data saturation was achieved. Further, the variety of interview techniques responded to participants' preferences.

\section{Conclusion}

Our findings suggest that communicating clinical information is significant in building shared situational awareness. Behaviors such as the use of self-talk, closed-loop communication, and overhearing conversations help teams to build shared situational awareness. Such behaviors allow team members to coordinate actions with others better, broadening the expertise and sphere of attention and reducing fixation. Thus team members are enabled to perform their roles in parallel and strategize accordingly. Any associated deficits in selectively attending to messages, transmitting information to the appropriate personnel, seeking additional information from key personnel, and 
eliciting a useful response, compounds the potential for adverse patient outcomes.

\section{Acknowledgments}

The research team is grateful to the staff working in the operating rooms at the study site for their willingness to participate. The team also wishes to thank Anne McMurray, Professor Emeritus, for her thoughtful critique of an earlier manuscript. BG gratefully acknowledges the financial assistance of the Griffith University New Researcher Grant and the Research Centre for Clinical and Community Innovation.

\section{Disclosure}

The authors report no conflicts of interest in this work.

\section{References}

1. World Health Organisation. New checklist to help make surgery safer. Press Release: New Checklist to Help Make Surgery Safer. Geneva, Switzerland: World Health Organisation; 2008.

2. Joint Commission on Accreditation of Health Care Organizations. Sentinel events trends reported by year. Joint Commission on Accreditation of Health Care Organizations; 2008. Available from: http://www.jointcommission.org/sentinel_event.aspx. Accessed January 31, 2013.

3. Gawande A, Zinner M, Studdert D, Brennan T. Analysis of errors reported by surgeons at three teaching hospitals. Surgery. 2003;133:614-621.

4. Gandhi T. Fumbled handoffs: one dropped ball after another. Ann Intern Med. 2005;142:352-358.

5. de Vries E, Ramrattan M, Smorenburg S, Gouma D, Boermeester M. The incidence and nature of in-hospital adverse events: a systematic review. Qual Saf Health Care. 2008;17:216-223.

6. Vincent C, Neale G, Woloshynowych M. Adverse events in British hospitals: preliminary retrospective record review. BMJ. 2001;322: 517-519.

7. Leape L, Berwick D. Five years after to err is human: What have we learned? JAMA. 2005;293:2384-2390.

8. Gillespie B, Chaboyer W, Longbottom P, Wallis M. The impact of organisational and individual factors on team communication in surgery: A qualitative study. Int J Nurs Stud. 2010;47:732-741.

9. Amalberti R, Auroy Y, Berwick D, Barach P. Five system barriers to achieving ultrasafe health care. Ann Intern Med. 2005;142:756-764.

10. Gilson RD. Situation awareness [Special issue]. Hum Factors. 1995;37(1)3-4.

11. Endsley M. Toward a theory of situational awareness in dynamic systems. Hum Factors. 1995;37:37-64.

12. Davies J. Team communication in the operating room. Acta Anaesthesiol Scand. 2005;49:898-901.

13. Tenney Y, Pew R. Situation awareness catches on: What? So what? Now what? Reviews of Human Factors and Ergonomics. 2006;2:1-34.

14. Wright M, Endsley M. Building shared situation awareness in healthcare settings. In: Nemeth $\mathrm{C}$, editor. Improving Healthcare Team Communication: Buliding on Lessons from Aviation and Aerospace. Hampshire, UK: Ashgate; 2008.

Journal of Multidisciplinary Healthcare

\section{Publish your work in this journal}

The Journal of Multidisciplinary Healthcare is an international, peerreviewed open-access journal that aims to represent and publish research in healthcare areas delivered by practitioners of different disciplines. This includes studies and reviews conducted by multidisciplinary teams as well as research which evaluates the results or conduct of such teams or
15. Flin R, Maran N. Identifying and training non-technical skills for teams in acute medicine. Qual Saf Health Care. 2004;13Suppl 1:i80-i84.

16. Mathieu J, Heffner T, Goodwin G, Salas E, Cameron-Brown J. The influence of shared mental models on team process and performance. J Appl Psychol. 2000;85:273-283.

17. Morineau T, Morandi X, Le Moëllic N, et al. Decision making during preoperative surgical planning. Hum Factors. 2009;51:67-77.

18. Flin R, Youngson G, Yule S. How do surgeons make intraoperative decisions? Qual Saf Health Care. 2007;16:235-239.

19. Pauley K, Flin R, Yule S, Youngson G. Surgeons' intraoperative decision making and risk management. Am J Surg. 2011;202:375-381.

20. Mitchell L, Flin R, Yule S, Mitchell J, Coutts C, Youngson G. Thinking ahead of the surgeon. An interview study to identify scrub nurses' non-technical skills. Int J Nurs Stud. 2011;48:818-828.

21. Kitto S, Chesters J, Grbich C. Quality in qualitative research: criteria for authors and assessors in the submission and assessment of qualitative research articles for the Medical Journal of Australia. Med J Aust. 2008; 188:243-246.

22. Manias E, Street A. Rethinking ethnography: reconstructing nursing relationships. J Adv Nurs. 2001;33:234-242.

23. de Laine M. Ethnography: Theory and Applications in Health Research. Sydney, Australia: MacLennan and Petty; 1997.

24. DeSantis L, Ugarriza D. The concept of theme as used in qualitative nursing research. West J Nurs Res. 2000;22:351-372.

25. Tobin G, Begley C. Methodological rigour within a qualitative framework. JAdv Nurs. 2004;48:388-396.

26. Shih FS. Triangulation in nursing research: issues of conceptual clarity and purpose. J Adv Nurs. 1998;28:631-641.

27. Hazelhurst B, McMullen C, Gorman P. Distributed cognition in the heart room: How situation awareness arises from coordinated communications during cardiac surgery. J Biomed Inform. 2007;40:539-551.

28. Riley R, Manias E. Gatekeeping practices of nurses in operating rooms. Soc Sci Med. 2009;69:215-222.

29. Klein G. The strengths and limitations of teams for detecting problems. Cognition, Technology and Work. 2006;8:227-236.

30. Johanessen L. Maintaining common ground: an analysis of cooperative communication in the operating room. In: Nemeth C, editor. Improving Healthcare Team Communication: Building on Lessons from Aviation and Aerospace. Hampshire, UK: Ashgate; 2008.

31. Patterson E, Watts-Perotti J, Woods D. Voice loops: engineering overhearing to aid coordination. In: Nemeth $\mathrm{C}$, editor. Improving Healthcare Team Communication: Building on Lessons from Aviation and Aerospace. Hampshire, UK: Ashgate; 2008.

32. Endsley M. Toward a theory of situation awareness in dynamic systems. Hum Factors. 1995;37:32-64.

33. Riley R, Manias E. Governance in operating room nursing: nurses' knowledge of individual surgeons. Soc Sci Med. 2006;62:1541-1551.

34. Wiegmann D, ElBardissi A, Dearani J, Daly R, Sundt T. Disruptions in surgical flow and their relationship to surgical errors: an exploratory investigation. Surgery. 2007;142:658-665.

35. Healey A, Sevadalis N, Vincent C. Measuring intra-operative interference from distraction and interruption observed in the operating theatre. Ergonomics. 2006;49:589-604.

healthcare processes in general. The journal covers a wide range of areas and welcomes submission from practitioners at all levels, from all over the world. The manuscript management system is completely online and includes a very quick and fair peer-review system. Visit http://www.dovepress.com/testimonials.php to read real quotes from published authors. 